\title{
Initial Evidence for Self-Organized Criticality in Electric Power System Blackouts
}

\author{
B. A. Carreras \\ Oak Ridge National \\ Laboratory \\ Oak Ridge TN 37831 \\ carrerasba@,ornl.gov
}

\author{
D. E. Newman \\ Physics Dept. \\ University of Alaska \\ Fairbanks AK 99775 \\ ffden@uaf.edu
}

\author{
I. Dobson \\ ECE Department \\ Univ. of Wisconsin \\ Madison WI 53706 \\ dobson@oengr.wisc.edu
}

\author{
A. B. Poole \\ Oak Ridge National \\ Laboratory \\ Oak Ridge TN 37831 \\ pooleab@ornl.gov
}

\begin{abstract}
We examine correlations in a time series of electric power system blackout sizes using scaled window variance analysis and $R / S$ statistics. The data shows some evidence of long time correlations and has Hurst exponent near 0.7. Large blackouts tend to correlate with further large blackouts after a long time interval. Similar effects are also observed in many other complex systems exhibiting self-organized criticality. We discuss this initial evidence and possible explanations for selforganized criticality in power systems blackouts. Selforganized criticality, if fully confirmed in power systems, would suggest new approaches to understanding and possibly controlling blackouts.
\end{abstract}

\section{Introduction}

Electric power transmission networks are complex systems that are commonly run near their operational limits. Such systems can undergo non-periodic major cascading disruptions that have serious consequences. Individually, these disruptions or blackouts can be attributed to specific causes, such as lightning strikes, ice storms, equipment failure, shorts through untrimmed trees, excessive customer demand, or unusual operating conditions. However, an exclusive focus on these individual causes can overlook the global dynamics of a complex system in which repeated major disruptions from a wide variety of sources are a virtual certainty. Indeed, large scale disruptions can be intrinsic to the global system dynamics as is observed in systems displaying Self-Organized Criticality (SOC) [1]. A SOC system is one in which the nonlinear dynamics in the presence of perturbations organize the overall average system state near to, but not at, the state that is marginal to major disruptions. SOC systems are characterized by a spectrum of spatial and temporal scales of the disruptions that exist in remarkably similar forms in a wide variety of physical systems. In these systems, the probability of occurrence of large disruptive events decreases as a power function of the event size. This is in contrast to Gaussian systems in which the probability decays exponentially with event size. Therefore, the application of traditional risk evaluation methods to SOC systems is bound to underestimate the risk of large events.

It is difficult to directly determine whether a system has SOC type dynamics. However, we can explore the existence of correlations of events over long time scales to indicate whether the system has non-trivial complex dynamics with non-Gaussian properties. We also consider the probability distribution function of disturbance sizes.

\section{Detecting long time correlations in time series}

A time series is said to have long-range dependence if its autocorrelation function falls off asymptotically as a power law. This type of dependence is difficult to determine because the noise tends to dominate over the signal for long time lags. Over the last decade, several techniques have been developed to overcome this problem. One such technique, and chronologically the first one, is the rescaled range statistics (R/S statistics) proposed by Mandelbrot and Wallis [3] and based on a previous hydrological analysis by Hurst [4]; another is the scaled window variance technique [2].

The $R / S$ statistics or the scaled window variance technique considers blocks of $m$ successive points in the integrated time series and measure how fast the range or standard deviation of the blocks grows as $m$ increases. In the case of the scaled window variance technique, we begin by considering a time series $X \equiv\left\{X_{t}: t=1,2, \ldots, n\right\}$. We then construct the associated series of the Brownian motion, $Y \equiv\left\{Y_{t}: t=1,2, \ldots, n\right\}$; that is, the original series integrated in time: $Y_{t}=\sum_{0}^{t} X_{t}$. For the Brownian motion series $Y$ and for each $m=1,2, \ldots, n$, a new series $Y^{(m)} \equiv\left\{Y_{u}^{(m)}: u=1,2, \ldots n / m\right\} \quad$ is generated. The elements of this series are blocks of $m$ elements of 
the initial series; that is, $Y_{u}^{(n)}=\left\{Y_{u m-m+1}, \cdots, Y_{u m}\right\}$. We then calculate the standard deviation, $\sigma_{m}^{(i)}$, within each of the $n / m$ blocks of $m$ elements of this series, and after we average $\sigma_{m}^{(i)}$ over the $n / m$ blocks to obtain $\sigma_{m}=(m / n) \sum_{i=1}^{n / m} \sigma_{m}^{(i)}$.

It can be shown that in the case of a time series $X$ with an autocorrelation function that has an algebraic tail, the function $\sigma_{m}$ scales as $\sigma_{m} \propto m^{H}$, where $H$ is the Hurst exponent. For $1>H>0.5$, there are long-range time correlations, and for $0.5>H>0$, the series has longrange anticorrelations. If $H=1.0$, the process is deterministic. When the data is uncorrelated, the Hurst exponent is 0.5 .

A constant $H$ parameter over a long range of time lag values is consistent with self-similarity of the signal in this range [5] and with an autocorrelation function that decays as a power of the time lag with exponent $\beta=2-2 \mathrm{H}$. In comparison with the direct determination of the autocorrelation function or other techniques of calculating the value of $H$, the scaled window variance analysis is robust.

\section{Analysis of blackout data}

We define blackouts as disturbances of the power transmission system that cause loss of power to customers. Every year, the North American Electrical Reliability Council (NERC) publishes a documented list summarizing major disturbances [6]. They are of diverse magnitude and the causes vary. It is not clear how complete this data is, but it is the best-documented source that we have found for disturbances in the North American power transmission system.

We reviewed NERC records for the years 1994 through 1997 inclusive to determine the causes for recent large blackouts. The breakdown of the 106 major blackouts for 1994 through 1997 is shown in Table 1. All of the blackouts were centered on the transmission system. The average time to restore power was approximately 3 hours. The number of major blackouts each year ranged from 21 to 29 with an average of 27 .

Table 1. Causes of major blackouts by region 1994-97.

\begin{tabular}{l|crrrc} 
Cause & W & NE & MW & SE & Total \\
\hline Weather -25\% & 6 & 3 & 13 & 4 & 26 \\
Equipment fails-47\% & 26 & 15 & 6 & 3 & 50 \\
Human error-12\% & 4 & 5 & 4 & 0 & 13 \\
Vandalism-9\% & 4 & 4 & 3 & 0 & 11 \\
Low reserves-7\% & 0 & 5 & 1 & 0 & 6
\end{tabular}

In trying to understand the global dynamical properties of the North American power transmission grid, we have done a simple correlation analysis of the reported blackouts between January 1993 and July 1998. We constructed time series with the resolution of a day for the number of disturbances and for four different measures of the blackout size. The blackout size was measured by the energy unserved (MWh), the amount of power lost (MW), number of customers affected and the restoration time. (Energy unserved was calculated from the NERC data by multiplying the power lost by the restoration time.) For example, the time series for the power lost is shown in Figure 1.

To these five series, the number of blackouts plus the four measures of blackout size, we have applied the scaled windowed variance analysis technique and the $R / S$ statistics to determine their respective Hurst exponents $H$. Both methods give consistent answers. There is a clear range of more that one decade of time lags (from about 30 days to 500 days) over which the averaged $\sigma_{m}$ shows a clear power dependence on time. Figure 2 shows the averaged $\sigma_{m}$ as a function of time delay for the number of blackouts and the energy unserved. The Hurst exponents obtained by fitting $\sigma_{m}$ are shown in Table 2 .

Table 2. Hurst exponents of blackout numbers and sizes

\begin{tabular}{l|c}
\multicolumn{1}{c|}{ Time series } & Hurst exponent $H$ \\
\hline Number of blackouts & 0.52 \\
Energy unserved (MWh) & 0.70 \\
Power lost (MW) & 0.58 \\
Number of customers & 0.69 \\
Restoration time & 0.67
\end{tabular}

For the time series of the number of blackouts, the Hurst exponent is about 0.5 . This value indicates that there is no correlation between individual triggers of the blackouts. This is not surprising and is perhaps what could be expected from the variety of random causes for the blackouts.

On the other hand, the analysis of the time series of blackout sizes show a clear existence of long range time correlations, as indicated by the Hurst exponents greater than 0.5 in Table 2 . The time record considered has only 1920 points. To test the significance of the results for the blackout sizes, a random scrambling of the time series leads to $H$ exponents that range from 0.49 to 0.55 . They are well below the values obtained for the original records, which suggest that their deviation from 0.5 is significant. The sequences do not show any signs of periodicity that could contaminate the determination of $H$.

We also used the NERC data from January 1993 to July 1998 to estimate the probability distribution function of the blackout sizes. For example, Figure 3 shows the probability distribution of the energy unserved in the blackouts. The fitted line shows that the probability decreases with power -0.98 . For the number of customers affected, the probability distribution function decays as a -0.65 power and for the restoration time the power is 
-1.13. For each of these measures of blackout size, the probability falls off relatively slowly with the blackout size. Since the exponents are clearly above -2 , the variance of the blackout size is unbounded. Furthermore, since the powers are close to -1 , even the mean could be unbounded. These results imply that blackouts of the size of the full grid are possible.

\section{Possible explanation of power system SOC}

We suggest a qualitative account of the structure and effects in a large scale electric power transmission system which could give rise to SOC. The transmission system contains many components such as generators, transmission lines, transformers and substations. Each component experiences a certain loading each day and when all the components are considered together they experience some pattern or vector of loadings. The pattern of component loadings is determined by the power system operating policy and is driven by the aggregated customer demands at the substations. The power system operating policy includes short time frame actions such as generator dispatch as well as longer time frame actions such as improvements in procedures and planned outages for maintenance. The operating policy seeks to satisfy the customer demands at least cost. The customer demand has daily and seasonal cycles and a secular increase. Moreover, the patterns of customer demand change due to the evolution of bulk power markets and geographic shifts in population and industry.

Events are either the limiting of a component loading to a maximum or the zeroing of the component loading if that component trips or fails. Events occur with a probability that depends on the component loading. For example, the probability of transformer failure generally increases with loading. Another example is that an operator redispatching to limit power flow on a transmission line to its thermal rating could be modeled as probability zero below the thermal rating of the line and probability one above the thermal rating.

Each event is a limiting or zeroing of load in a component and causes a redistribution of power flow in the network and hence a discrete increase in the loading of other system components. Thus events can cascade. If a cascade of events includes limiting or zeroing the load at substations, it is a blackout. A stressed power transmission system experiencing an event must either redistribute load satisfactorily or shed some load at substations in a blackout. A cascade of events leading to blackout usually occurs on a time scale of minutes to hours and is completed in less than one day.

It is customary for utility engineers to make prodigious efforts to avoid blackouts and especially to avoid repeated blackouts with similar causes. These responses to a blackout occur on a range of time scales longer than one day. Responses include repair of damaged equipment, more fiequent maintenance, changes in operating policy away from the specific conditions causing the blackout, installing new equipment to increase system capacity, and adjusting or adding system alarms or controls. The responses reduce the probability of events in components related to the blackout, either by lowering their probabilities directly or by reducing component loading by increasing component capacity or by transferring some of the loading to other components. The responses are directed towards the components involved in causing the blackout. Thus the probability of a similar blackout occurring is reduced, at least until load growth degrades the improvements made. There are similar, but less intense responses to unrealized threats to system security such as near misses and simulated blackouts.

The pattern or vector of component loadings may be thought of as a system state. Maximum component loadings are driven up by customer demand trends via the operating policy. High loadings increase the chances of cascading events and blackouts. The loadings of components involved in the blackout are reduced or relaxed by the responses to security threats and blackouts. However, the loadings of some components not involved in the blackout may increase. These opposing forces driving the component loadings up and relaxing the component loadings are a reflection of the standard tradeoff between satisfying customer demands economically and security. The opposing forces apply over a range of time scales. We suggest that the opposing forces, together with underlying growth in customer demand and diversity give rise to a dynamic equilibrium and conjecture that this dynamic equilibrium is SOC.

Table 3. Analogy between power system and sand pile

\begin{tabular}{l|ll} 
& Power system & Sand pile \\
\hline system state & loading pattern & gradient profile \\
driving force & customer demand & addition of sand \\
relaxing force & response to blackout & gravity \\
event & limit flow or trip & sand topples
\end{tabular}

We briefly indicate the roughly analogous structure and effects in an idealized sand pile model which is expected to show SOC [7]. Consider a large, idealized sand pile which has grains of sand added at a continuously varying location. When the local maximum gradient gets too large, sand at that location is more likely to topple. Events are the toppling of sand and cascading events are avalanches. The system state is a vector of maximum gradients at all the locations in the sand pile. The driving force is the addition of sand, which tends to increase the maximum gradient, and the relaxing force is gravity, which topples the sand and reduces the maximum gradient. SOC is a dynamic equilibrium in which avalanches of all sizes occur and in which there are long time correlations between avalanches. The analogy between the sand pile and the power system is shown in Table 3. There are also some distinctions between the two systems. In the sand pile, the avalanches 
are coincident with the relaxation of high gradients. In the power system, each blackout occurs on fast time scale (less than one day), but the knowledge of which components caused the blackout determines which component loadings are relaxed both immediately after the blackout and for some time after the blackout.

\section{Conclusion}

The time series of blackout sizes presented above show long-range time correlations as well as power dependent tails in their distribution functions. These initial results suggest that the global dynamics of the power transmission system are those of a complex dynamical system. Such system may be close to a SOC system. The results are consistent with SOC, but there is not yet enough evidence to fully confirm this. Longer and more detailed records of blackouts would be helpful, as well as more refined methods to distinguish SOC dynamics from data.

The correlation results suggest that large blackouts are correlated with further large blackouts after a long time interval. In general, the SWV and R/S analyses presented here go beyond cumulative statistics such as the probability density function to reveal some temporal information about the system dynamics.

We have also suggested a qualitative description of the global dynamics of a large scale electric power system. These global dynamics are broadly analogous to the dynamics of an idealized sand pile model that is expected to show SOC. This outline of a possible explanation of $\mathrm{SOC}$ in a power system shows the opposing forces that could give rise to a dynamic equilibrium with $\mathrm{SOC}$ properties. The opposing forces are, roughly speaking, the trends in load demands weakening parts of the system and the responses to blackouts strengthening parts of the system. It is interesting to reflect that responses to a blackout are usually regarded as an outcome of a detailed investigation of particular blackout causes. However, the more global view suggested here sees responses to blackouts as an intrinsic part of the global system dynamics.

If electric power transmission systems are found to obey SOC like dynamics, this would have a number of important implications. First and perhaps most striking is the intrinsic unavoidability of cascading events in such a system when driven near its operational limits. When an event occurs the natural tendency is to focus on the cause of that event and try to prevent it from happening again. While often justified, this overlooks the fact that there will always be unforeseen events that will act as triggers. Reaction to a trigger which has already occurred will not impact another type of trigger and the potential severity of a resulting disruption depends less on the individual trigger (or sequence of events) then it does on the overall system state. This means that the global system state is at least as important in assessing a system's vulnerability to disruption as the state of some individual components.

On a more reassuring note, because of the apparently universal nature of SOC systems, we can learn a great deal about their dynamics from studying simple paradigmatic SOC models. Indeed, our qualitative description of SOC dynamics is a first step towards a more quantitative simulation model that captures the essentials of the global complex dynamics of the power system. The SOC or other complex dynamics in such a model would provide insights into global power system dynamics and allow more critical examination of explanations of SOC. Such a model could also serve as a test bed for developing methods for predicting and even controlling the overall system state to reduce the chances of large blackouts. One can even speculate about the possibility of determining statistical precursors to blackouts, which could allow real time corrections to prevent large blackouts.

Power transmission systems are large, complicated interconnected structures which underpin our society. Our initial results are suggestive of SOC in the global dynamics governing blackouts and we look forward to probing these complex dynamics further.

\section{Acknowledgments}

Part of this research has been carried at Oak Ridge National Laboratory, managed by Lockheed Martin Energy Research Corp. for the U.S. Department of Energy under contract number DE-AC05-960R22464.

\section{References}

[1] P. Bak, C. Tang, and K. Wiesenfeld, "Self-Organized Criticality: An Explanation of 1/f Noise," Phys. Rev. Lett., vol. 59 , pp. 381-4, 1987.

[2] M. J. Cannon, D. B. Percival, D. C. Caccia, G. M. Raymond, and J. B. Bassingthwaighte, "Evaluating scaled windowed variance methods for estimating the Hurst coefficient of time series," Physica A, vol. 241, pp. 606-626, 1997.

[3] B. B. Mandelbrot and J. R. Wallis, "Noah, Joseph, and operational hydrology," Water Resources Research, vol. \&, pp. 909-918, 1969.

[4] H. E. Hurst, "Long-term storage capacity of reservoirs," Trans. Am. Soc. Civil Eng., vol. 116, pp. 770, 1951.

[5] G. Samorodnitsky and M. S. Taqqu, Stable Non-Gaussian Random Processes: Stochastic Models with Infinite Variance New York: Chapman and Hall, 1994.

[6] Information on electric system disturbances in North America can be downloaded from the NERC website at http://www.nerc.com/dawg/database.html.

[7] P. Bak, "How Nature Works: The Science of SelfOrganized Criticality", Copernicus books, 1996. 


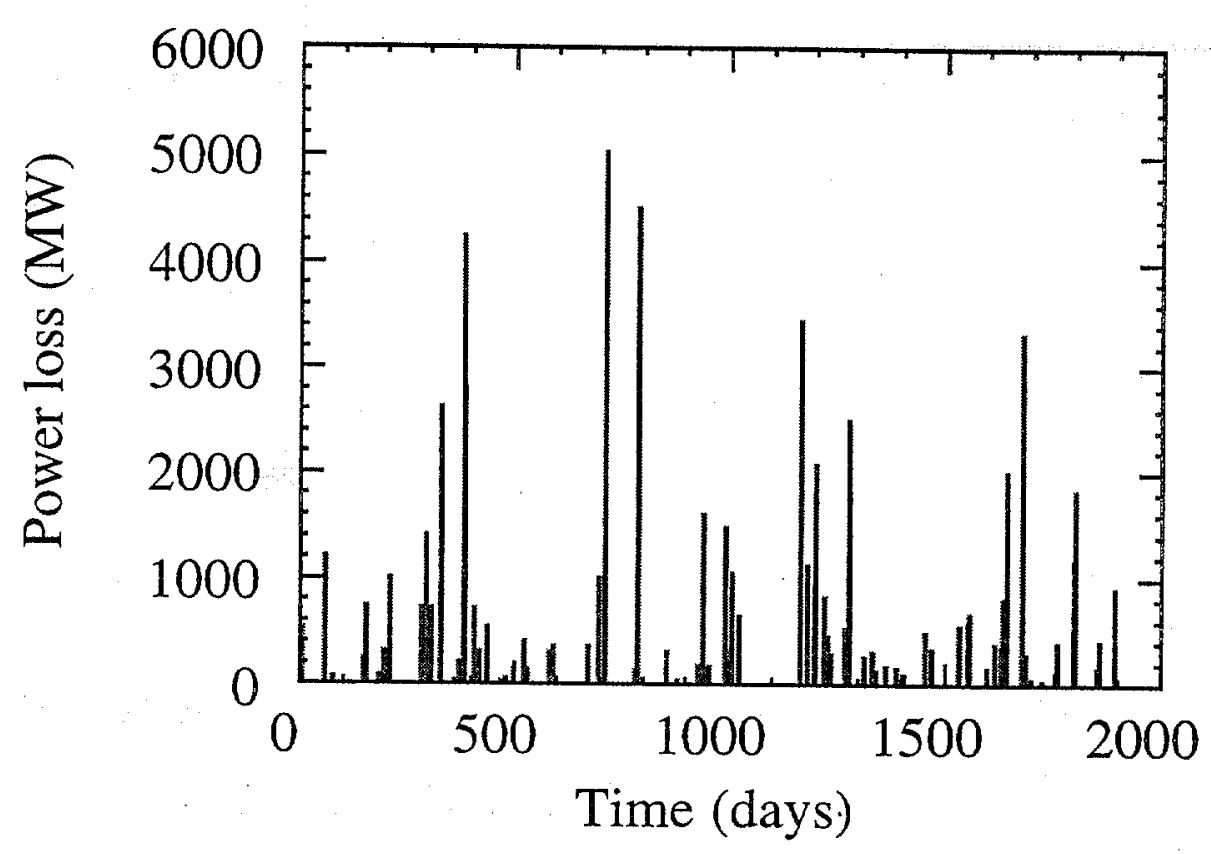

Figure 1. Power loss caused by blackouts in North America 1993-1998.

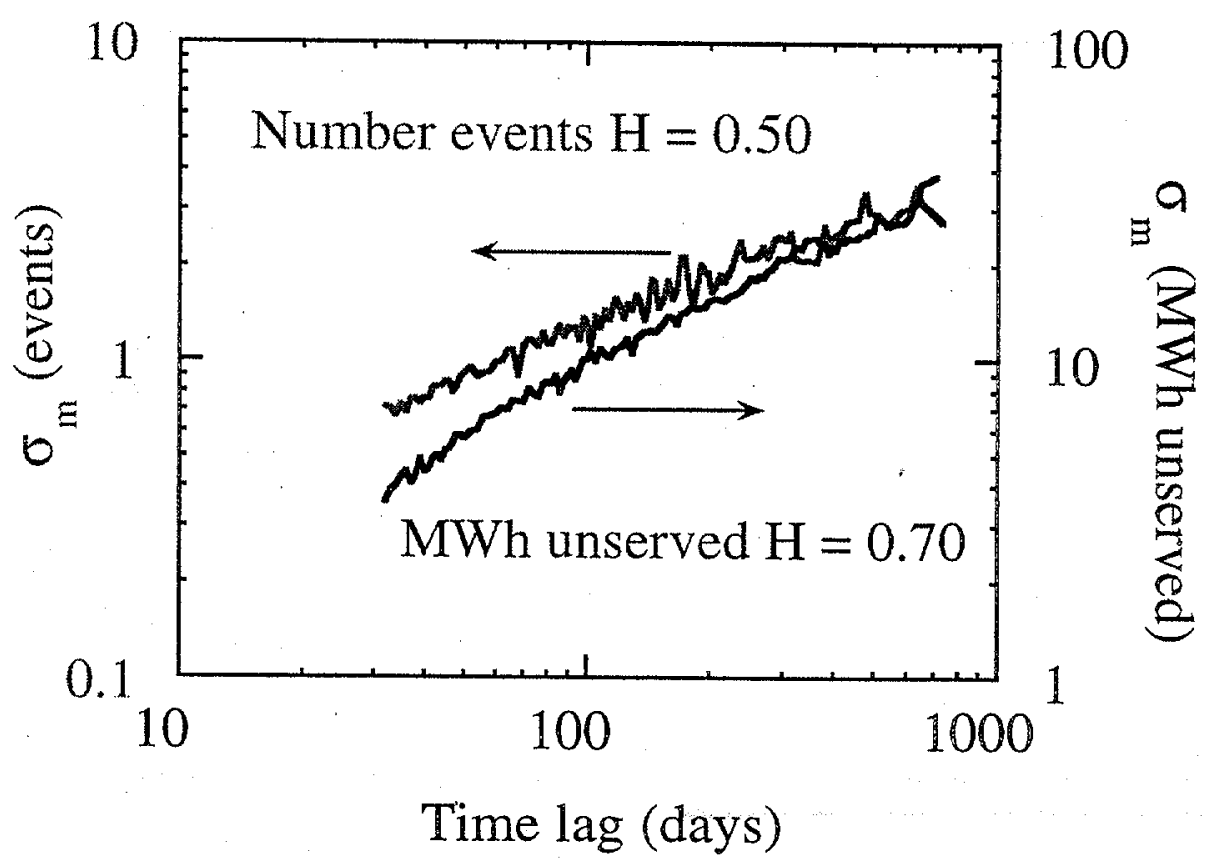

Figure 2. Scaled windowed variance analysis of the number of blackouts and energy unserved. 


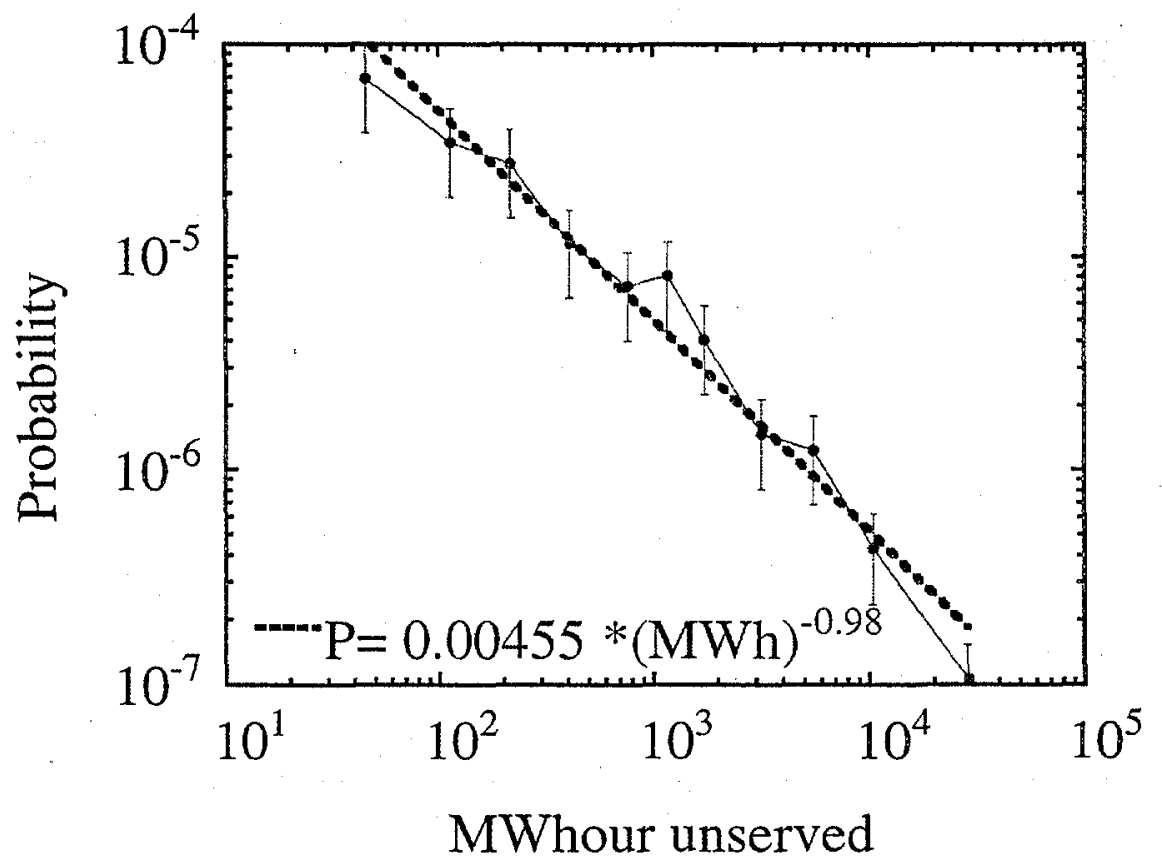

Figure 3. Probability distribution function of energy unserved for North American blackouts 1993-1998. 